\title{
Vocación médica en estudiantes de medicina de una universidad peruana
}

Medical vocation in medical students of a Peruvian university

\author{
Alberto Perales ${ }^{1}$, Elard Sánchez ${ }^{1}$, Alfonso Mendoza ${ }^{1}$, Charles Huamaní ${ }^{2}$ \\ ${ }^{1}$ Instituto de Ética en Salud, Facultad de Medicina, Universidad Nacional Mayor de San Marcos, Lima, Perú. \\ ${ }^{2}$ Instituto Nacional de Salud, Lima, Perú.
}

\begin{abstract}
Resumen
Objetivo: Evaluar las percepciones y desarrollo de la vocación médica en estudiantes de medicina de una universidad peruana. Diseño: Estudio cualitativo. Institución: Facultad de Medicina, Universidad Nacional Mayor de San Marcos. Participantes: Estudiantes de medicina. Métodos: Se realizó entrevistas semiestructuradas a 50 estudiantes de medicina, de primero a quinto año. Se indagó sobre: 1) definición de vocación, 2) definición de vocación médica, y 3) el desarrollo de su propia vocación médica. Principales medidas de resultados: Comparación de vocación médica entre los alumnos actuales y la de médicos ya realizados. Resultados: La mitad de los entrevistados fue de sexo femenino, edad promedio 21,7 años; $8 \%$ era hijo/a de médico; $82 \%$ era natural de Lima Metropolitana. Los estudiantes identificaron que la vocación médica tiene tres características: aspiración a ayudar, voluntad de servicio y espíritu de sacrificio. Sobre el desarrollo de su propia vocación, la decisión para estudiar medicina puede forjarse en la niñez temprana o en la adolescencia y, aún, ser incipiente al momento de ingresar a la Facultad de Medicina, siendo influenciada por diversas vivencias, dentro de las que destacan contactos con familiares médicos o con médicos en el curso de alguna enfermedad que los estudiantes padecieron. También existe influencia familiar e interés en relación al prestigio social. Conclusiones: Comparativamente, la vocación médica de los alumnos actuales y la de médicos ya realizados, muestran similitudes y diferencias. Entre los aspectos similares, un patrón de aspirante a médico altruista con motivaciones espirituales, económicas y sociales. En las diferencias, las motivaciones externas son más influyentes en los estudiantes actuales para iniciarse en la medicina así como una fascinación por el desarrollo tecnológico. Al respecto, se dan recomendaciones a los centros formadores.
\end{abstract}

Palabras clave: Vocación médica, estudiantes de medicina, características.

Abstract

Objectives: To determine perceptions and development of medical vocation in undergraduate students of a Peruvian University School of Medicine. Design: Qualitative study. Setting: Faculty of Medicine, Universidad Nacional Mayor de San Marcos. Participants: Medical students. Methods: Semi-structured interviews were applied to 50 medical students from first through fifth year, inquiring on: 1) Definition of vocation, 2) Definition of medical vocation, and 3) Student's vocational development. Main outcome measures: Comparison of medical vocation between current students and graduate phsysicians. Results: Half of the students were female, with mean age 21.7 years. Eight per cent were sons of physicians; $82 \%$ were born in Lima city. The students identified three characteristics in medical vocation: wish to help, will to serve, and disposition for sacrifice. In relation to his/her own vocational development, the decision to study medicine may begin at an early age or in adolescence, and even be emerging at the time of enrollment to the School of Medicine. Vocation is also influenced by diverse personal experiences mainly contact with physician's relatives or with physicians while suffering personal illness. There are also family influence and search for social prestige. Conclusions: Comparatively, medical vocation in undergraduate students and graduate physicians show similarities and differences. The expectation to be an altruistic physician with spiritual, economic and social motivations were common to both groups. Among the differences, the students showed a more external motivation to begin the career of medicine and fascination for technologic development. Some recommendations are given to teaching centers.

Keywords: Medical vocation, medical students, characteristics.

An Fac med. 2014;75(1):37-42

\section{INTRODUCCIÓN}

Este es el tercer trabajo de una línea de investigación sobre vocación médica ${ }^{(1,2)}$. En anterior informe habíamos descrito que esta puede comprenderse como un fenómeno viviente que depende, además de un factor individual (el interés de la propia persona), de un ambiente o entorno que estimule y sustente consistentemente su desarrollo ${ }^{(2)}$. La carrera médica es de alta exigencia, no solo porque implica una prolongada formación y adquisición de conocimientos científicos y habilidades profesionales, sino también, por el desarrollo de competencias cada vez más complejas. En tal sentido exige, aparte de una sólida vocación de servicio, de altas cualidades espirituales ${ }^{(3)}$.
Debido a las responsabilidades que la medicina implica, los procesos universitarios de selección de aspirantes a seguir la carrera han sido siempre los de mayor exigencia académica. En el Perú, sin embargo, particularmente en los últimos años, estos se han visto distorsionados por el rápido y desordenado incremento de facultades de medicina ${ }^{(4)}$, muchas de las cuales reducen en 
demasía los requerimientos de ingreso. Por otro lado, tales procesos de selección habitualmente preferencian el rendimiento académico del postulante, negligiendo evaluar la calidad de su vocación o de sus cualidades personales para ejercer la medicina ${ }^{(5)}$, siendo pocas las universidades que desarrollan un proceso de admisión altamente competitivo ${ }^{(6)} \mathrm{y}$ menos aún las que incluyen evaluación de actitudes.

En esta línea de reflexión, los hallazgos de nuestro estudio anterior ${ }^{(2)}$ nos permiten afirmar que, en el desarrollo de su vocación, además de motivaciones personales del aspirante a médico, existen otros factores externos que la influirán en el curso de su formación (tales como el entorno socio-profesional del estudiante, la influencia y ejemplo del cuerpo docente, el ambiente hospitalario, etc.) que, de estimularlos adecuadamente, reforzarán y consolidarán su vocación médica. Agregado a ello, y en virtud de las profundas transformaciones experimentadas por nuestra sociedad en las últimas décadas, con su inevitable impacto en las creencias, costumbres y valores de las nuevas generaciones, resulta legítimo pensar en la partcipación de otras motivaciones para escoger la carrera médica, cuya dinámica y peso sean, tal vez, radicalmente distintas de las tradicionales. Por ejemplo, estudios efectuados sobre el tema en otras realidades muestran que los estudiantes de medicina son influenciados, cada vez más, por intereses científicos y tecnológicos ${ }^{(7)}$, en lugar de por aquellos altruístas o sociales que solían ser prioritarios antaño ${ }^{(5)}$. En tal perspectiva, la vocación médica de los actuales estudiantes pudiera diferir de la descrita en nuestro trabajo previo, en los profesionales ya realizados ${ }^{(2)}$. Por tales razones, y considerando la vocación como variable importante para la elección de la carrera -problema sobre el cual no hemos encontrado estudios previos en el Perú en estudiantes de medicina-, hemos estimado importante llenar este vacío de conocimiento y continuar profundizando nuestra línea investigando sobre este tema, centrándonos, en esta oportunidad, en la po- blación estudiantil de la Facultad de Medicina de nuestra universidad.

\section{MÉTODOS}

El presente es un estudio cualitativo que incluye una muestra de 50 estudiantes, de primero a quinto año, de la Escuela de Medicina de la Universidad Nacional Mayor de San Marcos (FMUNMSM). La investigación se llevó a cabo en el segundo semestre académico del año 2009.

Se eligió a los estudiantes de la FMUNMSM debido a que suelen provenir de diversas regiones geográficas del país y pertenecen a una variada extracción socioeconómica. La selección de los estudiantes fue a través de un muestreo no probabilístico intencional, con la finalidad de maximizar la heterogeneidad en cuanto a las variables sexo y rendimiento académico. Para esta última, se solicitó a la Escuela de Medicina de la FM-UNMSM las notas del primer semestre. De la lista de notas se seleccionó al azar 10 estudiantes de cada año académico, 5 de rendimiento que los situaba en el tercio superior y 5 en el tercio inferior, a quienes se invitó a participar previa firma del consentimiento informado. En aquellos casos en los que el alumno o la alumna no aceptó, se invitó al siguiente en la lista de méritos académicos, hasta completar el número de 10 por año.

Para la recolección de datos se realizó entrevistas semiestructuradas, audiograbadas, con una duración promedio de 40 minutos, en los ambientes de la FM-UNMSM, a cargo de un mismo entrevistador. Se eligió la metodología de entrevistas en profundidad en lugar de grupos focales dado que se deseaba recoger experiencias concretas personales y con mayor detalle, así como percepciones negativas sobre la carrera que quizás pudieran no ser expresadas en grupo.

Las entrevistas fueron organizadas en tres apartados con preguntas norte. A cada entrevistado se le planteó: $1^{\text {o) }}$ Cómo definía el concepto de vocación; 2o) Cómo definía vocación médica; y, finalmente, $3^{\text {}}$ ) Que narrara libremente el proceso de su experiencia personal en cuanto al inicio y otros factores relacionados al desarrollo de su propia vocación médica. Se eligió estas preguntas sobre la base de aquellas utilizadas para la exploración conceptual y vivencial de la vocación en el trabajo anterior ${ }^{(2)}$.

Además de generar estos apartados, el entrevistador solo respondió a dudas conceptuales. El término de cada apartado se definió cuando el entrevistado ya no tenía más que contestar, o si se detectaba redundancias en sus respuestas.

Con la transcripción de las entrevistas se estableció unidades de significado y categorías que conformaron los temas a interpretar cualitativamente, identificándose las variables asociadas a la generación y desarrollo de la vocación. Las expresiones de los estudiantes y médicos en cada apartado son presentadas de forma comparativa, con citas resumidas. Los datos referentes a edad, sexo y otros de carácter demográfico son presentados en frecuencias relativas y absolutas calculadas en Excel 2010.

Con relación a los aspectos éticos, previamente se solicitó los permisos respectivos a las autoridades de la $\mathrm{Fa}$ cultad de Medicina y de la Escuela de Medicina. El proyecto fue presentado y aprobado por el Comité de Ética de Investigación de la Facultad de Medicina de San Fernando, de acuerdo a las normas que rigen la investigación científica en seres humanos. Finalmente, se solicitó el consentimiento informado a cada participante y tras la firma del documento, se procedió a la entrevista. Las fichas de recolección de datos fueron codificadas para asegurar la confidencialidad de los participantes.

\section{RESULTADOS}

\section{Características de la población}

El 50\% de los entrevistados fue de sexo masculino. La edad promedio fue 21,7 años, con un rango de 18 a 45 años. El 
8\% era hijo/a de médico; $82 \%$ informó ser natural de Lima Metropolitana; $51 \%$ era el mayor de los hermanos, teniendo entre 1 a 10 hermanos. El promedio de intentos de ingreso a la universidad fue 3,5 (rango de 1 a 8 veces). Solo el 6\% había ingresado al primer intento. En lo referente a los padres, en 21 casos $(44,7 \%)$ el padre, y en 18 casos $(38,3 \%)$ la madre tenían profesión universitaria.

\section{Definición de vocación}

El 86\% de los estudiantes indicó que la 'vocación' es una extensión de la aspiración o inclinación a lograr algo. Sin embargo, el 40\% complementó esta explicación refiriendo que ella debía incluir 'satisfacción' y 'tener aptitudes'. Todos los casos consideraron que se trata de una decisión voluntaria (anotamos ejemplos breves y pertinentes tomados de las entrevistas).

- Aspiración: “...aspiración de una persona para alcanzar sus metas y que satisface su visión de la vida". FAS, varón, 4to año.

- Satisfacción: "Es el impulso que te lleva a hacer algo sin estar pensando en una retribución, porque el hacerlo te da satisfacción y llena tus expectativas de vida, puede estar involucrada con la felicidad de una persona porque uno se entrega y hace lo mejor que pueda por el gusto que siente al hacerlo". NCI, mujer, 2 do año.

- Aptitudes: "La aptitud que una tiene para ciertas cosas, una predisposición para dedicarse a realizar una actividad y que en esto influye los gustos que uno tiene, las expectativas, los ideales". JCA, mujer, ler año.

\section{Definición de vocación médica}

La definición de 'vocación médica' se mantiene como una extensión de la aspiración; sin embargo, persigue como objetivo el 'servicio' a la población y destacándose un reconocimiento al 'sacrificio' que implica cursar la profesión.

- Aspiración: "aspiración de una persona por ganar conocimiento y brindarlos para lograr la salud tanto de la comunidad, la nación y las personas que lo rodean". FAS, varón, 4to año.

- Servicio: "ayudar a las personas en el ámbito de la salud, de estar a su servicio, sin necesidad de tener algo a cambio". CBN, mujer, ler año.

- Sacrificio: "...requiere sacrificio, tiempo y entrega, es prácticamente casarse con la medicina". ELC, varón, 1er año.

\section{Desarrollo de su propia vocación médica}

Decisión para estudiar medicina. Los estudiantes refieren dos momentos, en particular, en los que se inicia el deseo de estudiar medicina: en la niñez temprana (sin factores asociados evidentes de modo tal que asumen que siempre la tuvieron) y en la adolescencia, a partir de experiencias que serán descritas en el siguiente apartado (tabla 1).

- "Siempre he querido ser médico...". FAS, varón, 4to año.

- "Desde el colegio, uno es más consciente de su realidad y me fui dando cuenta de la situación de las personas y que podía hacer algo por los otros... en $3^{\circ}$ de secundaria resolví estudiar medicina". IBQ, mujer, 4to año.

- "Siempre estuvo en mí el deseo de ayudar. A los 5 años quería ser policía, a los 8 años quería ser veterinaria y a los 12 años me incliné por la medicina". MMT, mujer, 1er año.

La decisión de estudiar medicina fue, con frecuencia, voluntaria, lo que no significa que no estuviera influenciada por determinadas vivencias.

- “...nadie me presionó. En la TV veía casos de pobreza y me preguntaba cómo se curaban. Yo quería ser médico porque quería ayudar a los demás. Eso me viene desde pequeña, yo quería ser médica, jugaba a ser médica". RSL, mujer, 1er año.

- "...mis padres siempre conocían mis notas, mis profesores les decían que estudiase medicina, pero yo les daba la
Tabla 1. Edad de inicio y características asociadas al desarrollo de la vocación para estudiar medicina.

\begin{tabular}{lcc} 
& Casos & Porcentaje \\
\hline Edad de inicio (años) & & \\
\hline a a 6 & 4 & 8 \\
7 a 11 & 14 & 28 \\
12 a 16 & 23 & 46 \\
$\geq 17$ & 9 & 18 \\
\hline Familiar médico & & \\
\hline Sí & 19 & 38 \\
Influencia familiar & & \\
\hline Sí & 19 & 38 \\
Padre & 6 & 31,6 \\
Madre & 4 & 21,1 \\
Ambos & 4 & 21,1 \\
Otro familiar & 5 & 26,3 \\
\hline
\end{tabular}

contra en mi casa porque quería tomar mi propia decisión". VBA, mujer, 4to año.

El 40\% refirió la existencia de una experiencia favorable relacionada con algún médico, sea por un familiar, con el médico de cabecera o en razón de tener un problema de salud. Sin embargo, solo el $12 \%$ señaló un 'evento motivador', que provino de contactos que podríamos denominar rutinarios.

a. Contacto con familiares médicos:

- "Desde chiquito veía a mi tío, médico traumatólogo, contar lo que hacía y eso me gustó". DFP, varón, 3er año.

- “... a los 4 años presencié la operación de cesárea de mi hermana que mi padre la hizo". RNR, mujer, 4to año.

- "Un tío materno es médico y cuando yo era chico lo admiraba". NEL, mujer, 1er año.

b. Contacto con médicos y experiencia de enfermedad

- "comenzó por la admiración que yo tenía en los médicos porque de niño era muy enfermizo, me impresionó el conocimiento y el buen trato que me daban". FAS, varón, 4to año. 
- "Nació de pequeño, cuando sufría de asma, me llevaban al hospital y veía en Emergencia la realidad de necesidad de atención médica, la desesperación de las familias, ahi pensaba que de grande quería servir y mejorar. Eso hizo nacer en mí el deseo de ser médico". CPT, varón, 3er año.

Influencias para estudiar medicina. Las influencias para estudiar medicina provinieron de la familia o de vivencias personales. La influencia familiar no siempre fue positiva. En ocasiones se dio una confrontación con la familia, habiendo quienes no deseaban que el hijo (a) estudiara medicina, en particular cuando el familiar era médico. No se identificó un patrón sobre quién era la persona que ejercía mayor presión para estudiar medicina.

- "Mifamilia me decía que debía pensar en otra carrera por lo difícil y por el tiempo que lleva". FAS, varón, 4to año.

- "Sobre todo por la influencia de mi mamá y mi tía materna, ellas tomaban la iniciativa y a las visitas les decían: <ella va a ser médico>". CBN, mujer, 1er año.

- "Fue un poco influenciada porque desde pequeña mi abuelo materno me decía que le gustaría tener un médico, por ello me interesé, y poco a poco me fui interesando por ayudar a las personas, por estudiar biología". CGB, mujer, 5to año.

- "Mi padre quiso ser médico pero no pudo por razones económicas, ese deseo me lo narró con pasión, me hacía ver la maravilla de la relación médicopaciente, eso me causó interés". MPF, varón, 5to año.

Las vivencias personales que influyeron para estudiar medicina se dieron en dos áreas: la social y la científica. Entre las sociales se mencionaron actividades de promoción social o de ayuda, y entre las científicas, aquellas vinculadas a los cursos de biología y otros afines a temas médicos apreciados en la televisión.

- "Por parte de mis amigos se hacían campañas de ayuda -navideñas, por ejemplo- y uno se sentía bien de ver que la gente se sentía mejor". IBQ, mujer, 4to año.

- "Lo decidí en el colegio porque me di cuenta que me agradaban los cursos de ciencia y me agradaba bastante ayudar a los demás en lo que se pudiera". JCA, mujer, 1er año.

- "Influyó mucho los programas en TV de contenido médico y eso hizo que me gustara la idea de ser médico". MMT, mujer, 1er año.

En relación a otro tipo de influencias o intereses, es importante subrayar que, con frecuencia, los encuestados no privilegiaron un interés económico más allá de lo razonable. Ellos destacaron ser conscientes que la profesión de médico no generaría demasiados recursos.

- "La carrera me daría cierta comodidad económica, no bastante dinero, pero lo suficiente para llevar una vida tranquila”. DCM, varón, 4to año.

- “... a los 16 años recién me di cuenta que no quería ganar dinero por ganar sino que hay que ayudar a los otros, no fue una cosa incrustada desde niña". GMG, mujer, 2do año.

Sin embargo, sí estaban interesados en el prestigio social que brinda el ser médico, significando con ello tanto una búsqueda de reconocimiento como del respeto de los pacientes.

- “...siempre he admirado la posición social que tiene el médico, su estatus social es alto por el altruismo en relación con otras carreras". FAS, varón, 4to año.

- "Si uno hace bien las cosas y si eres líder, los demás te reconocen y te dan poder". CBN, mujer, 1er año.

\section{DISCUSIÓN}

Los estudiantes de medicina consideran que la vocación médica tiene tres características: aspiración a ayudar, voluntad de servicio y espíritu de sacrificio. Pero, es evidente que en su desarrollo se conjugan múltiples factores. Entre estos se encuentran los vivenciales, a través de las experiencias de asistir o presenciar actos médicos; los familiares, con o sin presión; los sociales, como el prestigio; los más espirituales, como aquellos relacionados con el exclusivo deseo de ayudar al prójimo; y la influencia de los medios de comunicación masiva, especialmente televisiva, sobre temas de salud. La definición de vocación y las motivaciones para su desarrollo, en forma general, no se alejan de las descritas en profesionales que han logrado su plena realización ${ }^{(2)}$. Sin embargo, guardan con ellas algunas diferencias importantes: 1) cuando se da el caso, la influencia de eventos médicos no se da comúnmente a través de una situación excepcional, sino que es mas bien la resultante de una serie de interacciones con figuras médicas de un modo podría decirse rutinario; 2) existe una influencia importante proveniente de la televisión, a través de series médicas; y, 3) son pocos los que describen un llamado más propiamente de su mundo interior, siendo lo más común la influencia de situaciones o personas estímulo del entorno externo.

Este último aspecto no es negativo, dado que los procesos de maduración nos permiten construir una personalidad a partir de necesidades y deseos reales o de fantasías, proceso en el que se van descartando algunas situaciones que dejan de agradarnos, o por el surgimiento de otros intereses. Ello ha sido descrito por H. Ginsburg ${ }^{(8)}$, quien postulaba que existían, en secuencia, las siguientes fases: de fantasía, de escuela y la realista. Esta diferencia entre los estudiantes comparativamente con los profesionales de años anteriores (2) se explicaría, además, por la diferencia generacional, que en promedio es de 50 años. Hipotéticamente hablando podría señalarse que las generaciones actuales, sometidas al impacto del acelerado desarrollo científico-tecnológico, sufren de una sobrecarga informativa y quizá destinen menor tiempo a la exploración y al cultivo de la propia interioridad. Estas diferencias entre la importancia del mundo interior, en contraste con el énfasis en la influencia 
del mundo externo que encontramos en un sector importante de la generación actual de alumnos, podría explicar algunas diferencias con los resultados de nuestro anterior estudio en médicos de generaciones anteriores ${ }^{(2)}$. Tal como lo describe John A. Mackay ${ }^{(9)}$ en su tesis, la vocación responde a una misión específica en la vida, que se interioriza tras varios años de reflexión. Llegar a tal nivel de maduración implica varios años de experiencia, por lo que pareciera hipotéticamente comprensible que en aquellos jóvenes estudiantes que sean menos introspectivos, los factores externos adquieran mayor relevancia; mientras que los profesionales nos expresan sus opiniones actuales, que presentan más factores internos, y que pueden haber variado respecto a sus etapas de estudiantes.

Con relación a la influencia de la televisión, ella puede vincularse a los cambios sociales y económicos vividos ${ }^{(10)}$. Mientras que los profesionales de antaño pueden haber sido influidos por un entorno social más duro y conflictivo, donde la apreciación de inequidades y de los problemas de salud pública era más palpable; los actuales estudiantes de medicina podrían experimentar esa influencia más que en el plano real en un entorno más bien virtual, a través de programas televisivos que aborden problemas médicos, a pesar de que en teoría provienen de estratos socioeconómicamente bajos ${ }^{(11)}$, aun cuanto no de extrema pobreza. Ello estaría en relación a las etapas iniciales de la vocación, en concreto a la etapa de 'fantasía' propuesta por $\mathrm{H}$. Ginsburg ${ }^{(8)}$, motivada por hechos no siempre reales o, en todo caso, con una fuerte carga de ficción, presenciados en la televisión, pero que satisfacen sus deseos y motivaciones intrínsecas.

En lo referente a los aspectos comunes, se encuentran razones espirituales, científicas y socioeconómicas que no son únicas en los estudiantes y profesionales evaluados, sino que se comparten con estudiantes en varias culturas ${ }^{(7,12)}$. Siguiendo la clasificación de Missenard, publicada por Jeammet ${ }^{(13)}$, en ella se encuentra el deseo de ayuda como una motivación de carácter más espiritual, el ego adiuvans del que habla Laín Entralgo ${ }^{(14)}$, al lado del afán de conocimiento y dominio de las leyes de la naturaleza, el ego sapiens, sin el cual no podría ayudarse realmente al paciente, debiendo por tanto, ya en el ejercicio de la profesión, articularse competencia técnica y voluntad de ayuda, conjunción necesaria que se halla en el núcleo de la vocación médica, la misma que debe reforzarse a través de todo el proceso formativo. Un fin menos noble pero no menos importante es el deseo de ganar dinero, siempre y cuando no se desvincule de los otros objetivos para no caer en la cosificación de la persona humana y convertirla solo en un objeto de lucro. Por último, relacionado con el propósito de obtener estatus y prestigio social, habría que considerar lo que el mismo Laín Entralgo denomina el ego fungens, refiriéndose al deber del médico que se desempeña como funcionario dentro de una institución pero que, como en el caso de las motivaciones antes expuestas, no debe anteponerse a la consideración, respeto y cuidado de la vida y la salud de las personas. Algunos de estos aspectos pueden confluir en un mismo estudiante, lo cual es consistente con otros estudios y revelaría el proceso de maduración de su vocación ${ }^{(5)}$.

Sobre la edad de inicio de la vocación para ser médico, tampoco existe un patrón definido. A pesar que se señala que el desarrollo de la real vocación es un proceso que se va construyendo, tanto algunos profesionales (2) como algunos estudiantes, señalan un origen 'muy precoz' o en la niñez temprana, lo que se contrapone a la vocación adquirida -en algunos casos- luego de iniciada su formación ya en la facultad de medicina. Este aspecto no es fácil de describir. En un estudio previo, en España, se señala que los estudiantes de medicina adquieren su vocación más tempranamente que sus pares de otras escuelas, debido a motivaciones más altruistas ${ }^{(5)}$ En un estudio anterior nuestro, ya citado, en algunos profesionales se daba el caso de no encontrar factor alguno que explicara la razón por la que estudiaban medicina ${ }^{(2)}$; mientras que otros necesitan de consejería o vivir la experiencia de la impronta de figuras ejemplares, es decir, de docentes con un auténtico 'eros pedagógico' en una escuela que fomentaba un clima emocional de profundo respeto por el otro, de auténtico altruismo ${ }^{(10)}$. De allí la importancia de considerar que la consolidación de la vocación y las motivaciones actuales para estudiar medicina sean influenciadas por profesionales modélicos, quienes con su comportamiento ejemplar devienen una suerte de imago de identificación y, de este modo, al transmitir sus experiencias contribuyen a humanizar la formación de las futuras generaciones ${ }^{(10,15)}$.

Nuestro trabajo revela, asimismo,que la familia emerge como la institución en la cual, a partir de sus propios mitos o creencias y de las motivaciones o deseos propios de los jóvenes, va germinando o reforzándose la vocación de los mismos. Este fenómeno no es exclusivo de nuestro medio, sino que ha sido descrito en estudiantes de diversas culturas ${ }^{(5,7,12)}$. Tal influencia familiar se expresa de manera variada, unas veces a través del contacto con otros profesionales, otras transmitiendo el deseo de tener un hijo médico, o a través de la experimentación de sucesos vitales difíciles, u otros momentos personales críticos donde el médico aparece como la figura que brinda ayuda técnica, comprensión empática y consejo oportuno.

El estudio tiene como limitación no poder describir la vocación del estudiante de medicina al final de su carrera, pues a pesar de haber incluido estudiantes de los últimos años, es posible que sus intenciones cambien pronto al apreciar el mercado laboral o al realizar el internado o el servicio médico rural y urbano marginal de salud (SERUMS) y conocer más de cerca la diversidad de la especialización médica ${ }^{(3,12,16)}$. Tampoco es posible extrapolar nuestros resultados a toda la población de estudiantes de medicina del Perú, dado que los estudiantes de San Marcos tienen peculiares características socioeconómicas y personales. Cabe resaltar, sin embargo, 
que estos factores -en particular los socioeconómicos-si bien pueden afectar su rendimiento académico ${ }^{(11)}$, pueden servir también de aliciente para continuar estudiando medicina y superarse personal, profesional y socialmente.

En conclusión, los factores que generan la vocación para estudiar medicina no han cambiado sustancialmente entre las generaciones de profesionales y de estudiantes de medicina evaluadas. Si bien existen factores comunes, también se dan elementos singulares que motivaron o influyeron en su desarrollo. Entre los aspectos similares, se constata la presencia de motivaciones espirituales, económicas y sociales. Entre las diferencias, en los estudiantes actuales se evidencia una motivación más externa para iniciarse en la medicina y una fascinación por el desarrollo tecnológico. Por último, al igual que en la historia vocacional de los ya profesionales, algunos estudiantes no habían definido completamente su vocación por la medicina, mostrando que ella puede estar en desarrollo. Este aspecto debe ser tomado en cuenta al momento de plantear la posibilidad de incluir en los procesos de selección de medicina una evaluación vocacional, para no cometer el error de excluir a aquellos postulantes que aún están en trance de consolidar una vocación incipiente y que, debidamente orientados, podrían devenir profesionales a carta cabal.

Desde esta perspectiva, la conclusión que resalta de la presente línea de investigación realizada en tres estudios, es que la obligación de toda facultad de medicina no sería propiamente averiguar si el postulante tiene o no vocación médica; más bien debería asumir que está presente desde que el o la joven está haciendo el esfuerzo necesario para su ingreso, aunque aceptando que no en todos la vocación se halla en el mismo nivel de desarrollo. En otros términos, tal vez podría decirse que la responsabilidad de la entidad formadora al evaluar la vocación de los futuros médicos debería ser precisar en qué momento evolutivo se encuentra, para apoyarlos del modo más adecuado con el fin de que el estudiante pueda clarificar y definir sus aspiraciones y objetivos en una tarea entre interpretativa y deliberativa, como la que se da en el proceso conjunto de toma de decisiones inherente a la relación médico-paciente, o entre el tutor y el alumno, proceso cuya resultante conducirá a una vocación enriquecida, fortalecida y gradualmente consolidada.

\section{PALABRAS FINALES}

El principal obstáculo que hemos encontrado en el estudio de la vocación médica en estos tres informes ha sido la dificultad de aprehender de manera objetiva variables que algunos expertos consideran inasibles para la investigación científica (vocación, valores, vida interna, entre otros). Creemos que ese es el aporte que ofrece la metodología cualitativa, que no solo permite precisar el fenómeno de estudio a través de la descripción de sus características distintivas que apuntan hacia una inicial definición, sino también, generar nuevas hipótesis de estudio que, posteriores esfuerzos, sea de los mismos investigadores o de otros interesados en el tema, podrán profundizar como explicación del fenómeno. Lo importante, ahora, es decidir cómo utilizar el conocimiento ganado en la mejora de los procesos de selección de estudiantes de medicina y cómo optimizar la cultura organizacional de los centros formativos -la Facultad de Medicina y sus hospitales docentes-, para seguir estimulando y reforzando la vocación médica de los estudiantes a través de adecuadas experiencias formativas -que deben ser planificadas y organizadas como parte del plan de estudios y no dejarla a la causalidad espontánea- en bien de ellos mismos, de la sociedad a la que habrán de servir, de la institución formadora y, en última instancia, de la misma medicina peruana.

\section{AGRADECIMIENTOS}

A las autoridades del Vice-Rectorado de Investigación, de la Facultad y de la
Escuela de Medicina de la Universidad Nacional Mayor de San Marcos. A los estudiantes participantes que generosamente nos ofrecieron sus datos.

\section{REFERENCIAS BIBLIOGRÁFICAS}

1. Perales A, Mendoza A, Sánchez E. Vocación médica; necesidad de su estudio científico. An Fac med. 2013;74(2):133-8.

2. Perales A, Mendoza A, Sánchez E. Vocación médica en médicos de prestigiada conducta profesional. An Fac med. 2013;74(4):291-300.

3. Lifshitz A. La vocación de médico general. Gac Méd Méx. 2004;140(S1):S21-S22.

4. Berrios-Reiterer J. La creación de Facultades de Medicina en el Perú - 1998. Rev Med Hered. 1998;9(3):95-7.

5. Soria M, Guerra M, Giménez I, Escanero JF. La decisión de estudiar medicina: características. Educ méd. 2006;9(2):91-7.

6. Goic A. Proliferación de escuelas de medicina en Latinoamérica: causas y consecuencias. Rev Méd Chile. 2002;130(8):917-24.

7. Wierenga AR, Branday JM, Simeon DT, Pottinger A, Brathwaite B. Motivation for and concerns about entering a medical programme. West Indian Med J. 2003;52(4):304-310.

8. Ginsburg H, Opper S. Piaget's Theory of Intelectual Development.New Jersey: Prentice Hall Inc.; 1969.

9. Mackay J. El sentido de la vida. Pláticas a la juventud. Buenos Aires: La Aurora; 1947.

10. Rivero-Serrano O. IV. La vocación humanística: motivación permanente para el estudiante de medicina. Gac Méd Méx. 2004;140(1):55-7.

11. Jara D, Velarde H, Gordillo G, Guerra G, León I, Arroyo C, Figueroa M. Factores influyentes en el rendimiento académico de estudiantes del primer año de medicina. An Fac med. 2008;69(3):193-7.

12. Amin Z, Tani M, Eng KH, Samarasekara DD, Huak CY. Motivation, study habits, and expectations of medical students in Singapore. Medical teacher. 2009;31(12):e560-569.

13. Jeammet PH, Reynaud M, Consoli S. Psicología Médica. Rio de Janeiro: Masson; 1982.

14. Laín Entralgo P. El médico y el enfermo. Biblioteca para el Hombre Actual. Madrid: Edic. Guadarrama; 1969.

15. Hundert EM, Douglas-Steele D, Bickel J. Context in medical education: the informal ethics curriculum. Med Educ. 1996;30(5):353-64.

16. Mayta-Tristan P, Mejia CR, Riega-Lopez P, RojasMezarina L, Posso M, Mezones-Holguin E. Proyección de trabajo en el interior del país y factores asociados en médicos recién colegiados de Lima, Peru 2010. Rev Peru Med Exp Salud Publica. 2011;28(2):186-93.

Artículo recibido el 15 de diciembre de 2013 y aceptado para publicación el 10 de enero de 2014.

Financiamiento: Universidad Nacional Mayor de San Marcos, Código de Proyecto: 080120341.

Correspondencia:

Dr. Alberto Perales

Correo electrónico: perales.alberto@gmail.com 